\title{
Field Aligned Flows Driven by Neutral Puffing at MAST
}

\author{
I. Waters, ${ }^{1, \text { a) }}$ H. Frerichs, ${ }^{1}$ S. Silburn, ${ }^{2}$ Y. Feng, ${ }^{3}$ J. Harrison, ${ }^{2}$ A. Kirk, ${ }^{2}$ and O. Schmitz ${ }^{1}$ \\ 1) University of Wisconsin-Madison, Department of Engineering Physics, Madison, Wisconsin, \\ $U S A$ \\ ${ }^{2)}$ Culham Centre for Fusion Energy, Abingdon, Oxfordshire, United Kingdom \\ 3) Max-Planck-Institut für Plasmaphysik, Greifswald, Germany
}

(Dated: 7 March 2018)

Neutral deuterium gas puffing at the high field side of the Mega Ampere Spherical Tokamak (MAST) is shown to drive carbon impurity flows that are aligned with the trajectory of the magnetic field lines in the plasma Scrape-Off-Layer. These impurity flows were directly imaged with emissions from $\mathrm{C}^{2+}$ ions at MAST by Coherence Imaging Spectroscopy and were qualitatively reproduced in deuterium plasmas by modeling with the EMC3-EIRENE plasma edge fluid and kinetic neutral transport code. A reduced one-dimensional momentum and particle balance shows that a localized increase in the static plasma pressure in front of the neutral gas puff yields an acceleration of the plasma due to local ionization. Perpendicular particle transport yields a decay from which a parallel length scale can be determined. Parameter scans in EMC3-EIRENE were carried out to determine the sensitivity of the deuterium plasma flow phenomena to local fueling and diffusion parameters and it is found that these flows robustly form across a wide variety of plasma conditions. Finally, efforts to couple this behavior in the background plasma directly to the impurity flows observed experimentally in MAST using a trace impurity model are discussed. These results provide insight into the fueling and exhaust features at this pivotal point of the radial and parallel particle flux balance, which is a major part of the plasma fueling and exhaust characteristics in a magnetically confined fusion device.

PACS numbers: 52.25.Fi, 52.25.Jm, 52.30.Cv, 52.55.Fa, 52.65.Kj, 52.65.Pp, 52.70.Kz

\section{INTRODUCTION}

Understanding plasma sourcing by neutral particles and exhaust by parallel transport along magnetic field lines in the Scrape-Off Layer (SOL) is critical to obtaining density control in a magnetic confinement device. This challenge in a nominally axisymmetric tokamak is transformed into a three dimensional problem due to the application of Resonant Magnetic Perturbation (RMP) fields. These RMPs are necessary for control of transient heat and particles loads due to steep plasma edge pressure gradients and high local current densities [13]. It has been shown that RMP fields introduce a 3D structure to the SOL region [4], and that these open perturbed magnetic field lines can enter the formerly enclosed plasma region. This can lead to enhanced particle flux out of the plasma region by plasma flows along these perturbed field lines [5].

Understanding the physics of SOL flows driven by parallel pressure gradients [6] is thus a key element in understanding the physics of particle transport with RMPs. The parallel pressure gradients depend on the amount of particles injected into a given SOL flux bundle (via diffusive transport or neutral sourcing) compared to the sink terms in the flux bundle (from flows along the parallel direction to the divertor targets or radial losses in the perpendicular direction). The physics of this fundamental process defines the particle flux balance at the plasma edge and the SOL. By studying 3D flows in the

\footnotetext{
a)iwaters@wisc.edu; 3dpsi.engr.wisc.edu
}

SOL generally, any insights gained could then be applied to understand the impact of RMPs specifically. By analyzing a situation with toroidally symmetric magnetic fields, but three dimensional fueling features, the situation can be simplified and the core underlying physics can be isolated.

Coherence Imaging Spectroscopy (CIS) was available at MAST to measure impurity ion transport in the plasma edge and SOL. These SOL measurements combined with a specific neutral gas injection at the highfield side enabled a direct investigation of the link between local plasma sourcing on a magnetic flux tube and the parallel impurity flow. While the CIS measurements were made directly of impurity velocities, here, we study in depth the underlying change in the background deuterium plasma, and then take first steps to link these background flows with the impurity velocity.

In this paper, a comparison between these impurity ion measurements and 3D modeling with the EMC3EIRENE [7] plasma edge fluid and kinetic neutral transport code is shown and discussed. In Section 2 we discuss the experimental measurements, and introduce the EMC3-EIRENE code. In Section 3 the results from the EMC3-EIRENE models are shown. In Section 4 we present the most important underlying physics with a reduced one-dimensional model, we connect the underlying deuterium flows to impurity velocities, and discuss possible extensions to this work. Finally in Section 5 conclusions on the most important physics results from this effort are presented. 


\section{METHODS}

\section{A. Coherence Imaging Spectroscopy}

A CIS diagnostic was deployed on MAST to measure impurity ion flows in the SOL and divertor [8]. CIS is based on narrow-band imaging Fourier transform spectroscopy, which can provide time-resolved two dimensional imaging of emission line brightness, width and center wavelength over wide fields of view $[9,10]$. Flows are measured using CIS by measuring the Doppler shift of impurity ion emission lines along camera lines of sight. The large amount of spatial information provided by this technique makes it ideal for studying localized and dynamic phenomena in the plasma edge. The CIS instrument was used to study flows in both the main chamber SOL and divertor of MAST, using spectral lines for the $\mathrm{C}^{+}(\lambda=514.2 \mathrm{~nm}), \mathrm{C}^{2+}(\lambda=464.8 \mathrm{~nm})$, and $\mathrm{He}^{+}(\lambda=468.6 \mathrm{~nm})$ ions. In the studied MAST discharges, these were intrinsic impurities due to sputtering off graphite plasma facing components and Helium glow discharge wall conditioning. By imaging in the different impurity line emissions, flow structures at different depths of the SOL can be accessed, based on which emission lines are predominantly excited for a given temperature: flows deeper in the plasma were imaged with emissions from $\mathrm{He}^{+}$ions whereas in the outer SOL the $\mathrm{C}^{+}$ ions featured the highest impurity line emissivity. For this manuscript all CIS measurements shown are of the $C^{2+}$ ion emissions. Due to their brightness and location they best captured the particular phenomena of interest. While there is some evidence for these flows in the measurements of $\mathrm{C}^{+}$and and $\mathrm{He}^{+}$ions it is of lower quality for these measurements: the $\mathrm{C}^{+}$ion emissions are highly localized to only a few pixels near the centerstack, and the $\mathrm{He}^{+}$ion measurements are noisier and require longer integration times.

When observing $\mathrm{C}^{2+}$ ion velocities at the high field side (HFS) SOL near the midplane, a particularly striking observation was the formation of three dimensional structures in the carbon emissions under certain conditions. Specifically, when deuterium gas fueling from a valve at the inboard midplane was used a distinctive helical structure appeared around the center stack in the 2D flow images (as shown in Figure 1), indicating strong modification of the carbon flows on a particular flux tube compared to the neighboring plasma. This localization on a flux tube is shown by the calculated location of a field line overlain in dashed white in the image. When the distance from the gas valve to the plasma separatrixaccording to equilibrium reconstruction-was sufficiently small $(<2.5 \mathrm{~cm})$, such patterns were robustly observed to form, with such data obtained in 45 pulses. Velocity of the impurity species along the line of sight are shown in a color coded map with yellow/red indicating flows away from the camera and blue/violet indicating flow toward the camera. The gas inlet is located behind the centerstack, out of sight of the camera. Measured $\mathrm{C}^{2+}$



FIG. 1: Image of MAST discharge 28751 integrated from 0.124-0.129s with CIS diagnostic showing emissions from $\mathrm{C}^{2+}$ ions with color representing the measured line integrated velocity. Dashed line is an equilibrium reconstructed field line projected on the image showing alignment between $\mathrm{C}^{2+}$ flows and flux tube.

velocities were in the range of $5-25 \mathrm{~km} / \mathrm{s}$ and decrease as the structure extends up and down the center column, away from the source of neutrals. A detailed description of this technique and the specifications of this diagnostic can be found in [8].

\section{B. EMC3-EIRENE Modeling}

EMC3 is a fluid edge plasma code coupled to EIRENEa kinetic transport code for neutrals. This code solves a set of fluid equations accounting for the momentum and continuity of the bulk plasma, the energy transport of electrons, the energy transport of the bulk ions, and also includes a trace impurity model taking into account the continuity and a simplified momentum balance of the impurity species [7]. Anomalous cross field transport (perpendicular to the field lines) is captured using a particle diffusion coefficient $\left(D_{\perp}\right)$ for the bulk ions, and impurity ions, as well as separate heat diffusion coefficients $\left(\chi_{\perp}\right)$ for ions and electrons. These plasma fluid equations are then coupled to the EIRENE neutral transport code [11]. Neutrals are sourced based on ion deposition onto the plasma facing components as well as via neutral puffing sources. These neutrals are allowed to propagate through the plasma background until they are ionized. The energy loss and ion source of these ionization processes are then coupled to the plasma fluid equations of EMC3. The code is then run iteratively between EMC3 and EIRENE until a convergence is reached. 
While originally designed for inherently 3D magnetic systems (e.g. stellarators [12]), EMC3-EIRENE has also been used for 3D magnetic perturbations to largely axisymmetric systems (e.g. tokamaks with applied resonant magnetic fields $[6,13,14]$ ), asymmetric impurity seeding [15], localized heating by ECRH [16], and-of particular interest for this work-has the capability to include non-axisymmetric fueling sources. This flexibility is critical because toroidally asymmetric perturbations to the plasma (either in density, power deposition, impurity concentration, or even surface interactions) can lead to three dimensional structures-even without a 3D magnetic field.

A series of simulations was carried out that included the full toroidal and poloidal extent of the MAST edge plasma. Computational grids were built based on axisymmetric EFIT equilibria reconstructions of the upper disconnected double null MAST discharge 28980. A flexible grid generator [13], was then used to build a computational grid that encompassed the full 360 toroidal degrees of the tokamak in nine toroidal blocks. Neutral deuterium molecules (D2) were then thermally sourced from a single location on the center column at the midplane. Additional fueling via neutral recycling from plasma facing components and divertor targets was then self consistently adjusted to maintain a constant user defined density at the inner surface boundary of the modeling domain. Input power (1.35 MW) and inner simulation boundary density $\left(1.0 \times 10^{13} \mathrm{~cm}^{-3}\right)$ were held fixed while neutral puffing rates, and cross-field terms were varied. These inputs over predict the plasma temperature in the SOL that was measured by Thomson scattering, but serve as a baseline for qualitative modeling of the impacts of diffusion and puffing rates on the overall structure that is observed. Unless otherwise noted, simulations were carried out without parallel viscosity terms and with only the bulk deuterium plasma. Additional steps were taken to bring the simulation more in quantitative agreement with the experiment by lowering the input power (and thus the SOL temperatures) and including the carbon species in the EMC3-EIRENE modeling via wall sputtering, enabling a direct comparison between the CIS and EMC3-EIRENE modeling as in [17, 18]. This will be discussed in more detail in Section III C.

Important parameters such as plasma flow, temperature, plasma density, and plasma source density can be extracted along a field line to analyze the steady state behavior in the parallel direction. All fueling cases are characterized by a highly localized plasma source, andas shown in a single exemplary case in Figure 2-a rapid increase in parallel flow away from the source location in both directions along a field line. This is followed by a more gradual decay of flow further along the field line. The ionization of neutrals results in a local drop in plasma temperature and an increase in density which results in static plasma pressure $\left(p=n \times\left(k T_{e}+k T_{i}\right)\right)$ increasing dramatically (Figure 2.a) in the region of the D2 puff, resulting in flows along the field lines (Figure 2.b).



FIG. 2: Static plasma pressure (a) and parallel plasma flow (b) along a field line launched from immediately in front of the atomic deuterium puffing site $(\mathrm{Z}=0.0 \mathrm{~cm}$, $\phi=0.0, \mathrm{R}=19.7 \mathrm{~cm})$ and launched from $5 \mathrm{~cm}$ above puffing location $(\mathrm{Z}=5.0 \mathrm{~cm}, \phi=0.0, \mathrm{R}=19.7 \mathrm{~cm})$. Highly localized plasma pressure spike drives plasma flow away from the puff in the flux tube that directly intersects the gas puff, while the vertically neighboring flux tube remains relatively unchanged in plasma pressure and shows extremely weak flows.

This is in contrast to the neighboring field line (with an initial location displaced vertically along the centerstack) shown in red in Figure 2. By moving $5 \mathrm{~cm}$ up the centerstack and following a different field line there is no localized spike in plasma pressure, and correspondingly no acceleration of flows.

\section{RESULTS}

\section{A. Impacts of Different Neutral Puffing Rates in Deuterium Plasmas}

Initially, a fueling scan was carried out where the rate at which D2 was puffed into the plasma was varied from no neutral puffing, to a rate of $3.1 \times 10^{20}$ molecules/s. In the fueling scan scenarios, $D_{\perp}$ and $\chi_{\perp}$ were held fixed at $10000 \mathrm{~cm}^{2} / \mathrm{s}$ and $30000 \mathrm{~cm}^{2} / \mathrm{s}$ respectively. The density was held fixed at the inner simulation boundary, and the total ionization source from recycling was allowed to vary such that this boundary condition on the density was met. A poloidal slice through the deuterium plasma flow pattern up and down the centerstack at $\phi=0$ is shown in Figure 3 and is typical of these simulations. Here, a $1.55 \times 10^{19}$ molecules/s D2 puff at $\mathrm{R}=19.6$ $\mathrm{cm}$ and $\mathrm{Z}=0 \mathrm{~cm}$ drives a flow which can be seen in the major toroidal direction beneath the puffing location and opposite the major toroidal flow above the puffing location. It is apparent that the deuterium flow in the perturbed flux tube produces a pattern similar to what was observed with the CIS diagnostic [8].

To further investigate this helical phenomena, it is use- 


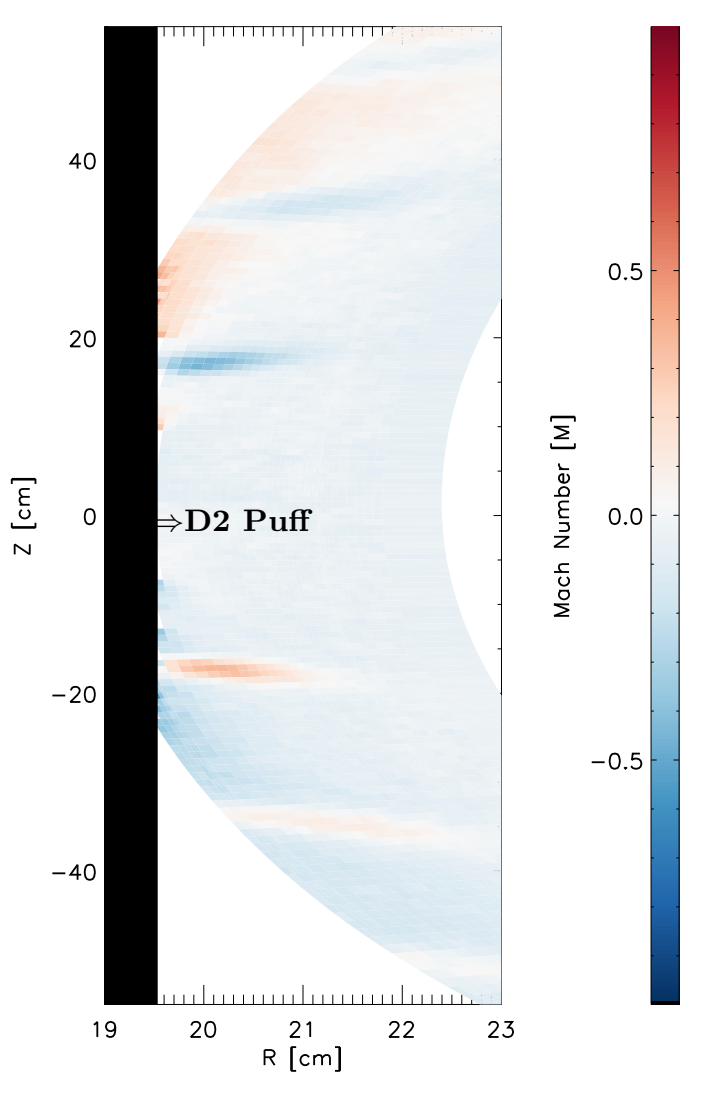

FIG. 3: Poloidal slice of deuterium Mach number in simulations zoomed in on HFS midplane. Figure is prototypical of flow pattern when point source of neutral particles is included in simulations at $\mathrm{Z}=0 \mathrm{~cm}$, $\mathrm{R}=19.6 \mathrm{~cm}$ (indicated on the figure). Flows shown in Mach number with the sign signifying either in the direction of toroidal field ( + in red) or against toroidal field direction (- in blue).

ful to look not only along a single field line as in Fig. 2, but along a set of radially neighboring field lines. This provides a cross section of the behavior across the flow channel from the area immediately in front of the neutral puffing location to areas deeper into the plasma. By launching field lines from sufficiently close positions (1 $\mathrm{mm}$ from point to point) and constraining our investigation along a field line to within 4 meters of the neutral puffing, geometric impacts of flux expansion can be ignored.

Figure 4 shows a series of these flux tubes for the four different fueling scenarios. The figure (top row) shows bulk plasma flow along the flux tube close to the centerstack, symmetric about the D2 puffing point (with the D2 puff located at $y=0$ in the figure). Flows are shown as normalized Mach number with positive flows in red and negative flows in blue. The intense localized plasma source caused by the neutral puffing drives strong flows. However, the peak flow location and overall structure of the flows isn't impacted by the puffing rate. Additional neutrals being introduced into the plasma only change the overall magnitude of the flows. The plasma source density (bottom row, shown in a log scale) shows similar qualitative consistency across the different fueling rates. In the region between 100 and $200 \mathrm{~cm}$ along the flux tube in either direction, a small background plasma source density can be seen in all four fueling scenarios. This results from the interaction of the SOL plasma particles with the centerstack (i.e. the self consistent sourcing of neutrals off the centerstack then being ionized by the bulk plasma). This sourcing does not lead to significant flows. In the three scenarios with neutral puffing an extremely peaked, localized, plasma source is seen with source densities 3-4 orders of magnitude higher than the more spread out source from recycling on the centerstack. It is in these cases that the local ionization drives plasma flow.

At rates higher than $3.1 \times 10^{20}$ molecules/s, stable solutions could not be found. The rate at which neutrals exited the centerstack in the experiments is not well bounded but some sources suggest $1.5 \times 10^{21}$ molecules $/ \mathrm{s}$ as a typical value [19], within an order of magnitude of our simulations. Despite this limitation, the 3D structures do form across an order of magnitude difference in neutral puffing rates and show similarities in structure with the experimentally observed $\mathrm{C}^{2+}$ ion flows.

\section{B. Impact of Cross Field Diffusion and Parallel Viscosity in Deuterium Plasmas}

Simulations were also carried out with different cross field diffusion rates $\left(D_{\perp}\left[\mathrm{cm}^{2} / \mathrm{s}\right]=1000,3000\right.$, and 10 000 , while $\left.\chi_{\perp}=3 D_{\perp}\right)$, but with neutral fueling and inner simulation densities kept constant (at $1.55 \times 10^{20}$ molecules/s and $1.0 \times 10^{13} \mathrm{~cm}^{-3}$ respectively). Field line profiles (starting at the point $\phi=0.0, \mathrm{Z}=0.0 \mathrm{~cm}, \mathrm{R}$ $=19.9 \mathrm{~cm}$, i.e. $3 \mathrm{~mm}$ in front of the puffing location) of flow are shown for the three diffusion values in Figure 5 . It can be seen that along the field line, the high diffusion coefficient case decays to $20 \mathrm{~km} / \mathrm{s}$ in half the distance than for the lowest diffusion coefficient. In general, the plasma equilibrium used for the modeling, and thus the EMC3-EIRENE simulation, does not have up-down symmetry. Thus, the field line is encountering different plasma parameters in the one direction (up the center column) versus the other direction (down the center column) and this difference in the background plasma may be responsible for the observed small asymmetries.

While these different diffusion coefficients change the parallel length scale of flows in the SOL, they also alter the temperature and density profiles of the edge plasma. In changing the diffusion coefficients from $D_{\perp}=1000$ $\mathrm{cm}^{2} / \mathrm{s}$ to $D_{\perp}=10000 \mathrm{~cm}^{2} / \mathrm{s}$ while maintaining a fixed density at the inner simulation boundary of $1.0 \times 10^{13}$ $\mathrm{cm}^{-3}$ at normalized poloidal flux of $\Psi=0.984$, the electron temperature at the inner boundary drops by a factor 


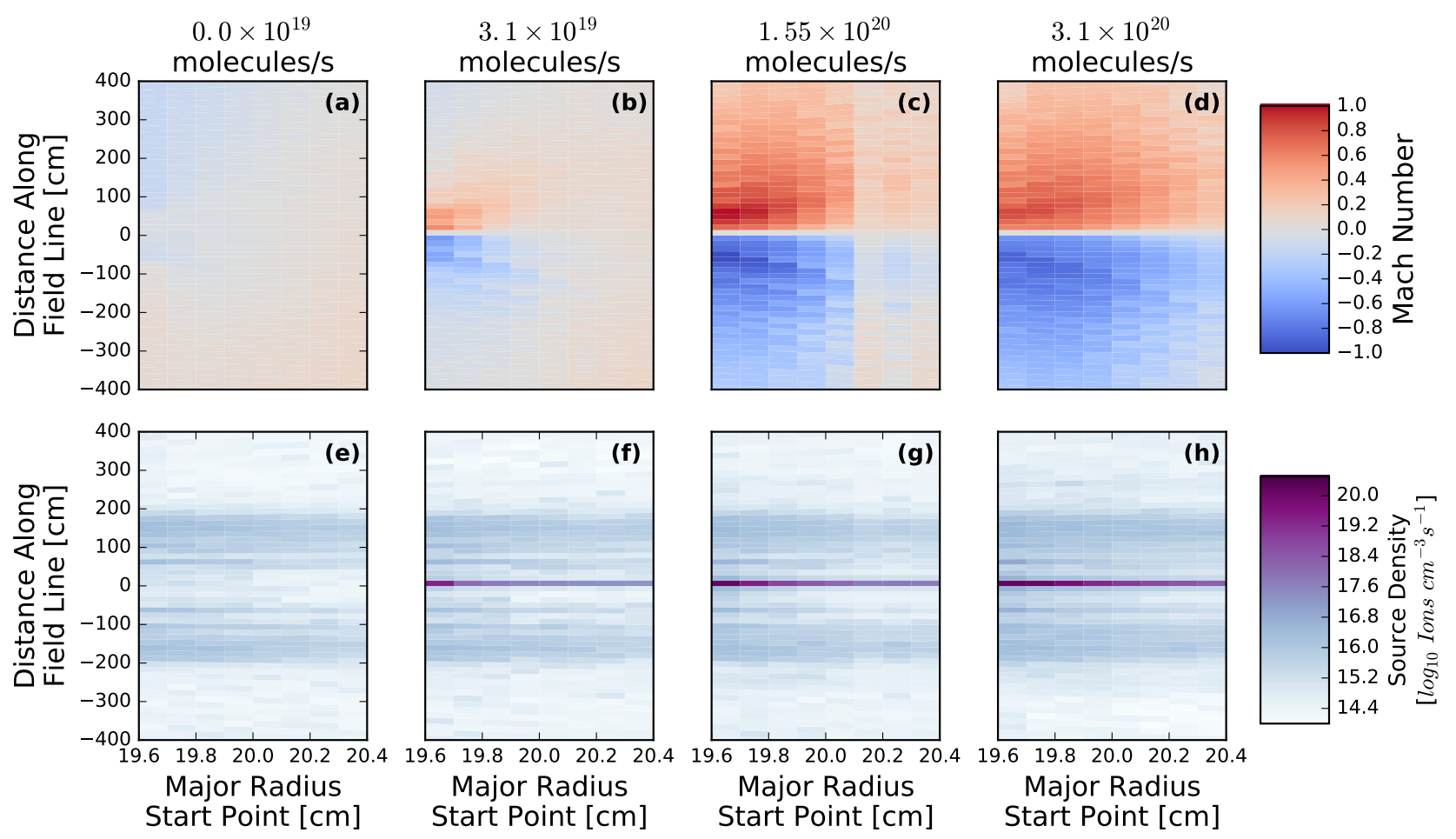

FIG. 4: Flow for 4 fueling scenarios in EMC3-EIRENE simulations along top row $(a=$ no neutral fueling, $b=3.1 \times 10^{19}$ molecules $/ \mathrm{s}, c=1.55 \times 10^{20}$ molecules $/ \mathrm{s}, d=3.1 \times 10^{20}$ molecules/s). Bottom row (e-h) shows source density.

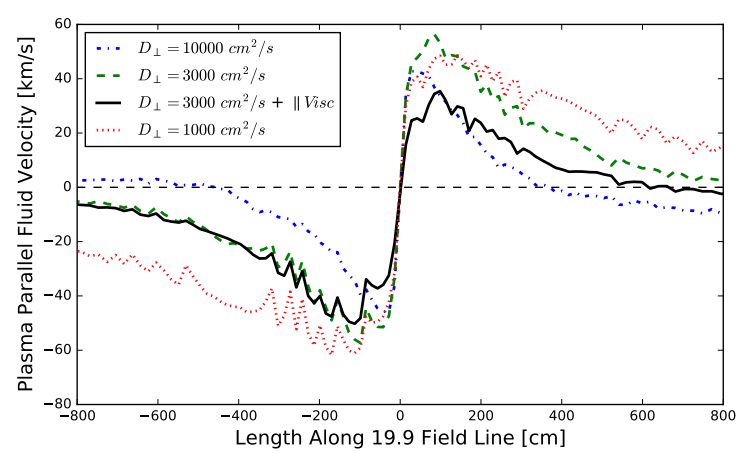

FIG. 5: Extracted profiles along the same field line in simulations with 3 different perpendicular diffusion coefficients $\left(D_{\perp}=3000 \mathrm{~cm}^{2} / \mathrm{s}\right.$ with and w/o $\|$-viscosity $)$ showing that low diffusion leads to more extended flows.

of 5 , from $412 \mathrm{eV}$ to $82 \mathrm{eV}$. This is a consequence of both the higher value of $\chi_{\perp}$ but also of the need in the simulation for more deuterium ionizations to fuel the plasma to achieve the same inner boundary density. That different diffusivity coefficients yield measurable flows of different length scales could be an important tool for plasma edge modeling. This could provide a check, independent of temperature and density measurements, to confirm that the cross field diffusion rates used for modeling a device are valid. While an intriguing possibility in theory, this has yet to be put into practice and would require additional work (discussed in Section IV) to link these diffusion dependent deuterium flows to the measured impurity species velocities.

Figure 5 also shows a case where the parallel viscosity term in the EMC3-EIRENE equations-typically omitted-is re-introduced into the simulation for the $D_{\perp}=3000 \mathrm{~cm}^{2} / \mathrm{s}$ case. We retain the same qualitative result for the flow with parallel viscosity included. However, the addition of this viscosity term leads to a slight decrease in the peak flow reached. There is also an asymmetry in the flow response to viscosity in the positive and negative direction along a field line. The addition of viscosity results in higher densities in the positive direction along the field line than in the negative direction and this is most likely responsible for the enhanced reduction in flow in this direction. The addition of the parallel viscous term acts to dampen the change of flow along a field line, thus static plasma pressure in the region of the source is enhanced by a factor of two. This increase in local pressure also results in a further perpendicular extension of the region of high pressure as shown in Figure 6 in which the static pressure is shown as a color map in the same domain as is used in Figure 4. It can also be seen that with parallel viscous terms acting on the plasma that the region of peak static pressure is no longer immediately in front of the atomic deuterium puff, but $2-3 \mathrm{~mm}$ into the plasma. The energy loss due to ionizations drives the 


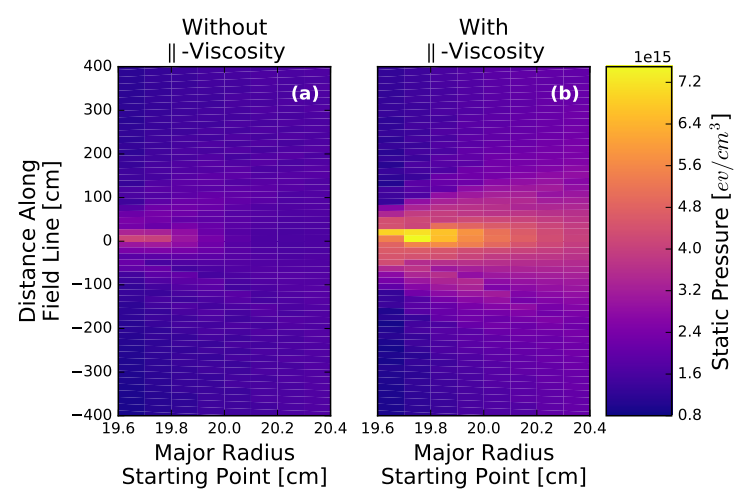

FIG. 6: Static plasma pressure along and across the effective flux tube in a $D_{\perp}=3000 \mathrm{~cm}^{2} / \mathrm{s}$ plasma without (a) and with (b) parallel viscous forces included in the simulation.

plasma temperature immediately in front of the puffing location down, which more than balances out the increase in plasma density. Only further into the plasma does the balance of particle sources and energy from the surrounding bulk plasma provide the maximum pressure.

\section{Inclusion of Carbon in Realistic SOL Conditions}

Motivated by the good qualitative agreement between the simulated flow structures in the pure deuterium plasmas and those imaged in the $\mathrm{C}^{2+}$ velocities, an additional pair of EMC3-EIRENE simulations was carried out: inputs were adjusted such that direct quantitative comparison between the simulations and experiment could be made. The main changes to the simulation inputs were that carbon impurity ions were included, and the input power was reduced to $450 \mathrm{~kW}$. The carbon impurities were sourced thermally off the vessel boundary and divertor targets via sputtering with a yield of one percent. The carbon ions could then be further ionized by the plasma or transported throughout the computational domain [7]. The trace impurity model in EMC3-EIRENE also includes power losses via radiation from the impurity species and this loss from the carbon ions, in tandem with the lower input power, produced a SOL that was within $10 \mathrm{eV}$ of the measured SOL temperature by Thomson scattering at the outboard midplane. The diffusion coefficients were set at $D_{\perp}=3000 \mathrm{~cm}^{2} / \mathrm{s}$ and $\chi_{\perp}=3 D_{\perp}$ for the background plasma, $D_{\perp}=3000 \mathrm{~cm}^{2} / \mathrm{s}$ for the impurity ions, and were constant throughout the simulation domain. The deuterium plasma flow pattern and $\mathrm{C}^{2+}$ densities are shown in Figure 7. It is clear that the overall pattern of deuterium flow in the perturbed flux tube is retained, and that this radial region of the plasma is in fact populated by $\mathrm{C}^{2+}$ ions as opposed to the higher or lower charge states. It should be noted that there is an asymmetry in the $\mathrm{C}^{2+}$ densities with higher densities seen on the lower half of the centerstack than the upper
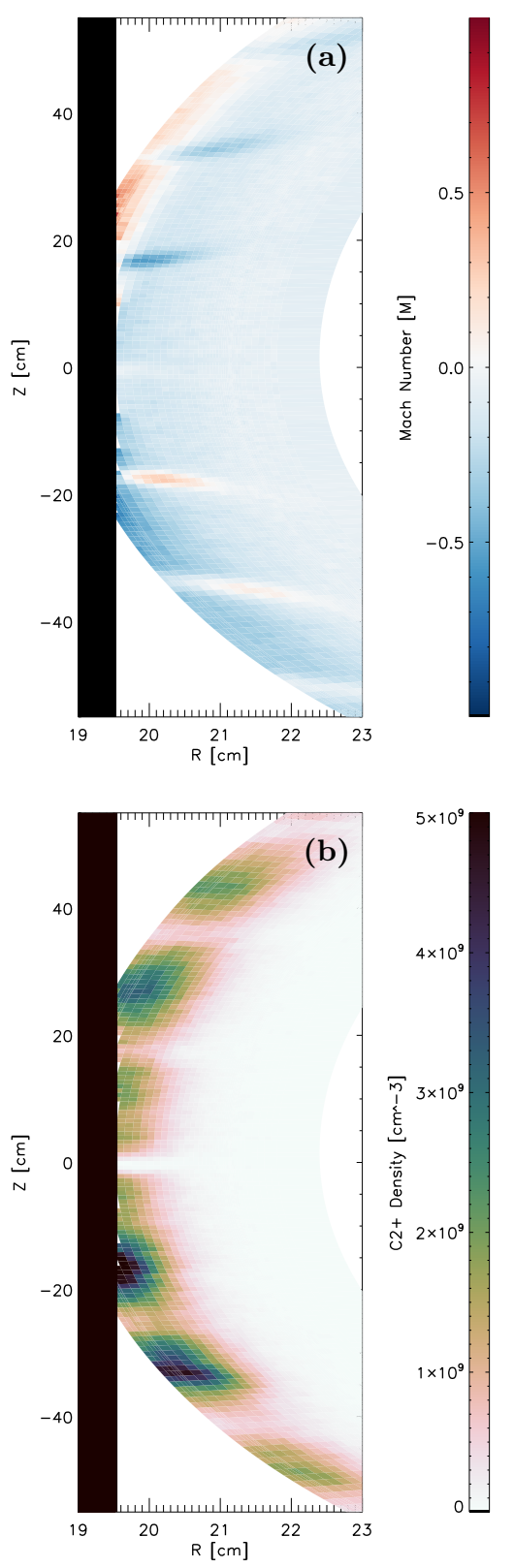

FIG. 7: Background deuterium flow (a) and $\mathrm{C}^{2+}$ density (b) for an EMC3-EIRENE simulation with input power of $450 \mathrm{~kW}, D_{\perp}=3000 \mathrm{~cm}^{2} / \mathrm{s}$, and $1.55 \times 10^{20}$ molecules $/ \mathrm{s}$ of neutral puffing.

half. Work towards extracting the velocities of the $\mathrm{C}^{2+}$ ions themselves is carried out below in Section IV C

\section{DISCUSSION}

\section{A. Flux Tube Analysis}

To better understand the features of the EMC3EIRENE results, comparative modeling has been carried 
out on a single (one dimensional) flux tube. This will provide a simpler case to link more fundamental physics to the complex 3D structures that are observed. We begin with a $1 \mathrm{D}$ isothermal ${ }^{1}$ fluid model (see [20] Chapter 10.2) for the ease with which it can be understood intuitively. We will then add additional physics as is necessary to qualitatively capture the features of interest seen in the EMC3-EIRENE results. To capture the qualitative behavior of the underlying functions a dimensionless formulation of the fluid model is presented below. We introduce the dimensionless quantities:

$$
\rho(\xi)=\frac{n(x)}{n_{b g}}, M(\xi)=\frac{u(x)}{c_{s}}
$$

And introduce the modeling parameter:

$$
\alpha_{S}=\frac{S_{0} L}{n_{b g} c_{s}}
$$

Where we define $n_{b g}$ as some prescribed background density, $c_{s}$ as the sound speed, $L$ as a perturbation scale length of the gas puff, $\xi=x / L$ as a dimensionless length parameter, and $S_{0}$ as a rate of the source strength.

We can then introduce a Gaussian source distribution to the flux tube using these dimensionless quantities and modeling parameters. The isothermal model can then be expressed as a coupled pair of particle (Eq. 3) and momentum (Eq. 4) conservation equations in an infinite flux tube:

$$
\begin{gathered}
\frac{d}{d \xi}(\rho M)=\alpha_{S} \frac{e^{\frac{-\xi^{2}}{2}}}{\sqrt{2 \pi}} \\
\frac{d}{d \xi}\left(\rho M^{2}+\rho\right)=0
\end{gathered}
$$

In this ideal model, a localized particle source leads to pressure gradient driven flows that continue along the flux tube to infinity in the absence of sinks. It is interesting to note that the value of $\rho(\xi=0)$ must be greater than the source parameter $\alpha_{S}$ or the flow solution will become undefined as $M$ exceeds 1 ([20] Chp. 1.8).

This is not what we see in the EMC3-EIRENE simulations when analyzing a single field line, and thus an additional physics term is required. We add expressions for cross field sinks into our 1-D characterization. The ansatz is made that the perturbed field line, where a plasma source is being added, interacts with a background field line with zero flow and a fixed density at the background value $n_{b g}$ through cross field diffusion. The perturbed field line then loses momentum and particles at some

\footnotetext{
1 The choice of an isothermal model may not seem appropriate given the energy sink that ionizing so many neutrals would produce. Later in this section we will check that this assumption is valid for our purposes.
}

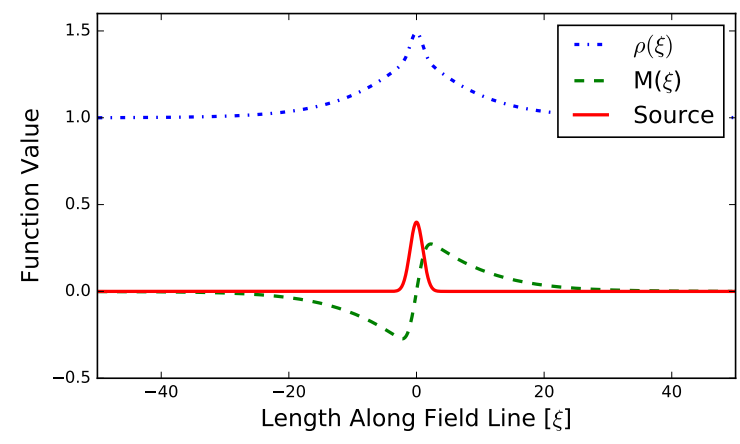

FIG. 8: Solving Eqs. 6 and 7 self consistently while imposing initial conditions on $\rho$ at $\xi=50$ and on $\mathrm{M}$ at $\xi=0$ in an infinite flux tube. Good qualitative agreement with Figure 2.

rate $D_{\perp}$, over some scale length $\delta$, and is proportional to the difference between the quantity (density or flow) on the two field lines. We can then define a diffusion timescale $\frac{D_{\perp}}{\delta^{2}}$ and parallel transport timescale $\frac{L}{c_{s}}$ with a dimensionless parameter:

$$
\alpha_{D}=\frac{D_{\perp} L}{\delta^{2} c_{s}}
$$

Which we then introduce back into the particle (6) and momentum (7) conservation equations:

$$
\begin{gathered}
\frac{d}{d \xi}(\rho M)=\alpha_{S} \frac{e^{\frac{-\xi^{2}}{2}}}{\sqrt{2 \pi}}+\alpha_{D}(1-\rho) \\
\frac{d}{d \xi}\left(\rho M^{2}+\rho\right)=-\alpha_{D} \rho M
\end{gathered}
$$

These equations can then be solved for given model parameters $\alpha_{D}$ and $\alpha_{S}$. We set $M(\xi=0)=0$ and at some distance from the stagnation point (many times the length scale of the perturbation, e.g. $\xi=50$ ), it is assumed that the density parameter $\rho(\xi)$ is equal to or very close to one (i.e. that the density returns to the background value at some very large distance from the perturbation). Numerical methods can then be used to find self consistent steady state solutions for $\rho(\xi)$ and $M(\xi)$ based on these initial conditions.

Figure 8 shows the results of this process for $\alpha_{S}=1$ and $\alpha_{D}=\frac{1}{5}$, with an initial value of $\rho(\xi=50)=1.0$. The shooting method is used from $\xi=50$ to determine an initial value of $M(\xi=50)$ to enforce the condition that $M(\xi=0)=0$. The resulting profiles for $\rho(\xi)$ and $M(\xi)$ are shown. The density peaks at some value above $\rho=1.0$ at the location of the source around $\xi=0$ before decaying back to $\rho=1.0$ symmetrically. The flow reaches a maximum (minimum) as it moves away from the source in the positive (negative) direction, and then exponentially decays along the field line until reaching equilibrium values. 
Having recreated the essential features of the EMC3EIRENE simulations along a field line in a general sense, we can then confirm that our input parameters are relevant to the MAST SOL simulations in a more absolute sense. To do this, we will determine what our modeling parameters of $\alpha_{S}$ and $\alpha_{D}$ would correspond to in real plasma parameters. Choosing a uniform plasma temperature $\left(\mathrm{T}_{e}=T_{i}\right)$ of $25 \mathrm{eV}$, a background density of $n_{b g}=1 \times 10^{13} \mathrm{~cm}^{-3}$, a length scale $\mathrm{L}=10 \mathrm{~cm}$ and a perpendicular scale of $\delta=.126 \mathrm{~cm}$, our modeling parameter of $\alpha_{S}=1$, will correspond to a source density of $S_{0}$ of $6.32 \times 10^{12}$ ions $\mathrm{cm}^{-3} \mathrm{~s}^{-} 1$. This corresponds to a peak source density of $2.52 \times 10^{12}$ ions $\mathrm{cm}^{-3} \mathrm{~s}^{-} 1$. Similarly $\alpha_{D}$ of $1 / 5$ is equivalent with these inputs to a $D_{\perp}$ of $10000 \mathrm{~cm}^{2} / \mathrm{s}$, aligned with the perpendicular diffusion coefficient used in some of our EMC3-EIRENE simulations. We can now verify if our isothermal assumption is reasonable by determining the perturbation in electron temperature required to provide the necessary energy to ionize this level of source. Using the equation for $q_{\|}$from [20] [Chpt. 9.6] and rearranging as shown below (Eqn. 8 ) we can directly calculate the required $\mathrm{dT} / \mathrm{dx}$.

$$
\frac{d T}{d x}=\frac{q_{\|}}{\kappa_{o e} T_{e}^{5 / 2}}
$$

It is then found that with the input parameters used here a gradient of $0.509 \mathrm{eV} / \mathrm{cm}$, or $5.09 \mathrm{eV}$ over the characteristic scale of the source distribution $\mathrm{L}=10 \mathrm{~cm}$ in our dimensionless formulation, would be sufficient to transport the necessary energy. Thus we can be confident that our simplistic 1D model will not be overly impacted by changes in temperature along the field line. A drop around the source region in temperature of $20 \%$ is coupled to an increase in density of nearly $50 \%$ and so the static plasma pressure will still rise locally, and still drive flows.

We can then use these values to translate back and forth between the EMC3-EIRENE simulations and the $1 \mathrm{D}$ model. We choose a flux tube in an EMC3-EIRENE simulation with a similar particle source (a flux tube 4 $\mathrm{mm}$ in front of the gas valve in the EMC3-EIRENE simulation fueled by $1.55 \times 10^{20}$ molecules/s peaks at a source density of $2.46 \times 10^{12}$ ions $\mathrm{cm}^{-3} \mathrm{~s}^{-1}$; this entire flux tube is shown in 4 (c) and (g)) and an equivalent diffusion coefficient. Figure 9 shows a direct comparison in source density, plasma density, and Mach number between the two models. While the magnitude of the two models is not exact, the qualitative features are retained. Both show the characteristic rapid peaking of Mach number and then decay on similar length scales. The two models differ most in the magnitude of the density perturbation where the 1D model shows a smaller density across the flux tube. The background density we've normalized to in our 1D model does not have a simplistic equivalent in the EMC3-EIRENE modeling so there is a higher and locally varying 'background density' in the EMC3EIRENE model These higher background densities, and even peaked densities in the vicinity of the fueling, would
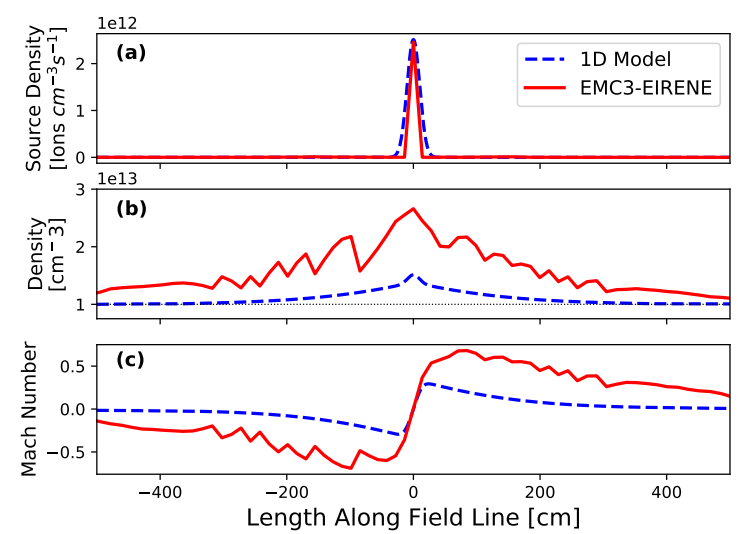

FIG. 9: Direct comparison between 1D model with $\alpha_{S}=1$ and $\alpha_{D}=\frac{1}{5}$ to EMC3-EIRENE simulation with neutral fueling of $1.55 \times 10^{20}$ molecules/s ( (c) and (g) in Figure 4) in source density (a), plasma density (b), and Mach number (c) along a single field line. While the EMC3-EIRENE simulation shows higher densities and Mach numbers, the two models show the same qualitative behavior.

then reduce cross field losses leading to higher densities along the field line of interest. These higher densities in the EMC3-EIRENE model lead to higher Mach numbers as the plasma moves away from the perturbation. Alternatively, the higher velocities in the EMC3-EIRENE simulation may be the result of the neighboring flux tubes, all of which also have neutral sources and flow. Thus the plasma in the EMC3-EIRENE may actually be gaining momentum from its neighbor on the puff side, and the drag resulting from cross field momentum losses will be reduced on the other side as this plasma is not stationary, as in our 1D model.

\section{B. Extracting Field Aligned Velocities from CIS Imagery}

The insights gained from detailed analysis of the modeled deuterium results-namely the characteristic decay of the plasma flow along a field line-motivates further analysis of experimental results. Since the CIS diagnostic measures emission line Doppler shift, the measurement gives the flow velocity component towards or away from the camera, and line-integrated along the diagnostic lineof-sight weighted by the local emission intensity. An estimate of the parallel flow speed can be extracted using an EFIT equilibrium and knowledge of the diagnostic viewing geometry. First, the sight-line vectors corresponding to each diagnostic pixel are obtained using the CalCam code $^{2}$, which allows spatial calibration of the camera by

\footnotetext{
${ }^{2}$ https://github.com/euratom-software/calcam
} 


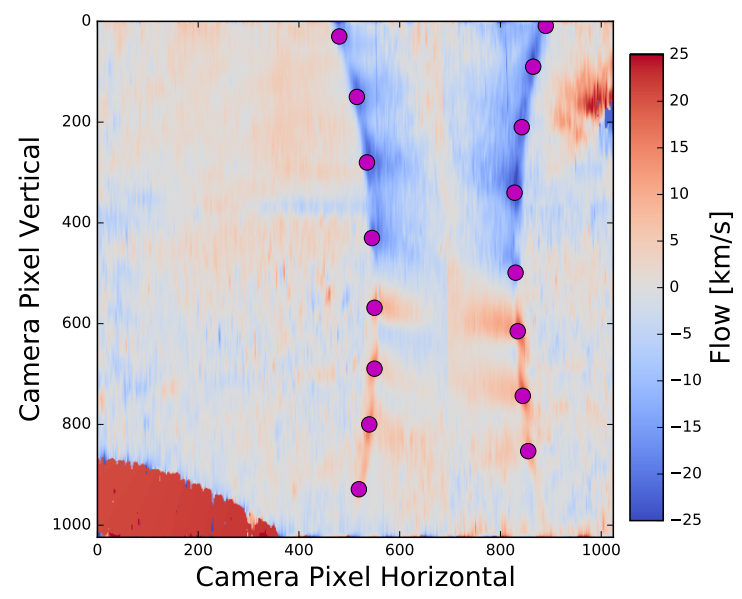

FIG. 10: Image from post processed CIS diagnostic showing flow speed in $\mathrm{km} / \mathrm{s}$ and with points indicating sampling locations for data in Figure 11.

matching features in images of the MAST vessel with features in a corresponding CAD model. Since the impurity line emission appears in a narrow shell in the SOL, and due to the horizontal viewing geometry of the diagnostic in this case, the measurement is well localized close to a curved plane where the camera sight-lines are tangent to the toroidal direction, known as the tangency plane. This allows estimation of parallel flow velocities without recourse to tomographic reconstruction techniques, which are required in more complex viewing and emission geometries such as for divertor measurements. It is assumed that the measured velocity is entirely in the parallel direction with respect to the magnetic field and that the contribution from cross field diffusion speeds are minimal. Then, the parallel flow at each point in the tangency plane can be estimated as $v_{\|} \approx v_{\text {measured }} / \mathbf{B} \cdot \mathbf{L}$, where $\mathbf{B}$ is a unit vector in the direction of the magnetic field and $\mathbf{L}$ is a unit vector in the direction of the camera's view direction, both taken at the tangency plane, for each pixel as shown in Figure 10. In this figure, the derived $v_{\|}$values for the tangency plane are shown. In order to obtain the variation in parallel flow along a specific field line, field line tracing is used to find the coordinates in the CIS image where a particular field line of interest intersects the tangency plane as shown by the overlaid points in Figure 10. This gives an estimate of the parallel flow at points along the field line spaced approximately half a toroidal turn along the field line.

Figure 11 shows the experimental flow values (taken from the points indicated in Figure 10) at their parallel distance along the flux tube from the neutral gas puffing site (found from field line tracing) in blue. These can then be compared with the EMC3-EIRENE simulations in Fig. 5. Good qualitative agreement is found between the two. Smaller diffusion coefficients produce bulk plasma flow structures that better match the overall shape of experimental measurements of the $\mathrm{C}^{2+}$ ion ve- locities. It should be noted that the magnitude of these velocities is not the same, nor should we expect it to be without fine tuning the model assumptions. The bulk plasma velocity will influence the impurity ion velocities but to what degree requires further analysis.

\section{Carbon Velocities in the EMC3-EIRENE Simulations}

Most of these simulations have been carried out with deuterium only, with the assumption that impurities will qualitatively follow the behavior of the bulk plasma flow. Analysis can be carried out on the simulation described in Section III C with realistic SOL temperatures and sputtered carbon impurities to verify that the carbon itself forms similar flow structures.

Within EMC3-EIRENE the simplified momentum balance equation (Eq. 6 in [7]) assumes a net force on the impurity ions of zero, which is consistent with neglecting the inertial terms in the momentum equation. The momentum conservation equation is then left as a balance of impurity momentum sources and sinks from pressure gradients, the ion and electron thermal gradients, the parallel electric field, and the frictional force between the impurity ions and the background plasma. We then find a momentum balance in the parallel direction of:

$$
\begin{array}{r}
m_{i m p} m_{i} \frac{v_{i m p}-v_{i \|}}{\tau_{s}}=-\mathbf{b} \cdot \nabla\left(n_{i m p} T_{i m p}\right)+ \\
0.71 n_{i m p} Z^{2} \mathbf{b} \cdot \nabla\left(k T_{e}\right)+n_{i m p} \beta_{i} \mathbf{b} \cdot \nabla\left(k T_{i}\right)+ \\
Z_{i m p} n_{i m p} e E_{\|}
\end{array}
$$

Where $\mathbf{b}$ is the unit vector of the B-field. No perpendicular forces have been included on the impurity ions (although anomalous perpendicular transport allows for the diffusion of impurities), the impurity temperature is assumed to be the same as the ion temperature, and the impurity species only impacts the background plasma through energy losses via radiation. The impurities do not apply forces back on the main plasma, and interactions between the different ionization states of the impurities are ignored [7]. Using the simplified momentum balance equation to find the velocity of the $\mathrm{C}^{2+}$ ions, in the same simulation shown in Section III C, along a field line launched from in front of the puffing location yields the green points shown in Figure 11. Not only are the qualitative features in agreement with the experimental observations, but quantitatively the numerical results are quite close as well. Similar comparisons between carbon impurity velocimetry measurements and EMC3-EIRENE simulations at the Large Helical Device $[17,18]$ also show good agreement, and together suggest that the EMC3EIRENE predictions of parallel impurity transport are largely valid, even without more complex transport processes included. 


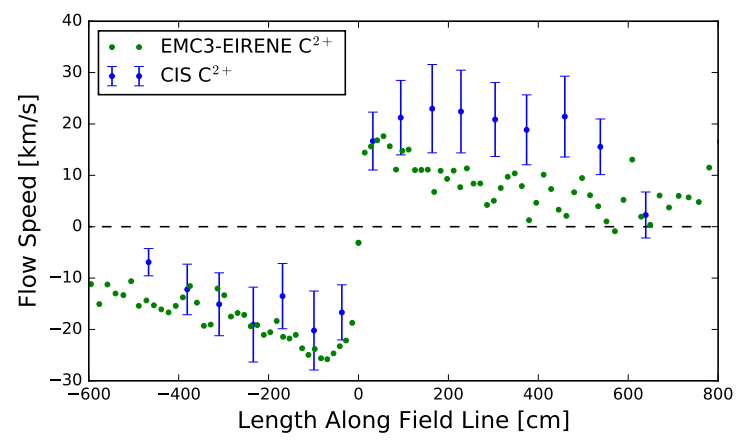

FIG. 11: The measured $\mathrm{C}^{2+}$ ion velocity along a field line measured at the points shown in Figure 10 shown in blue. Green points are the $\mathrm{C}^{2+}$ parallel ion velocities as extracted from EMC3-EIRENE simulations described in Section III C.

\section{Open Questions and Next Steps}

There are a number of factors that could impact the impurity transport and may explain discrepancies between the experimental results and the simulations. Of particular interest is the more poloidally diffuse emissions from the $C^{2+}$ ions in the CIS imagery as compared to the numerical results. Our simulations show flows that extend radially out from the centerstack and into the plasma. The CIS imagery show flows that stretch more up and down along a flux surface. Adjusting the perpendicular diffusion rate of the impurities may help provide better agreement [17]. Alternatively, line of sight integration intrinsic to the experiment may be the cause of the discrepancy. Use of a synthetic diagnostic to interpret the EMC3-EIRENE results could be used to test this. Efforts are underway to couple the EMC3-EIRENE code to the CHERAB code [21] for the development of a synthetic diagnostic for the MAST CIS system that will expand on existing EMC3-EIRENE synthetic diagnostic development [22]. We have also not yet addressed the role of bulk flows (without the neutral puffing perturbation) in either the experiment or the simulation and this could be a key component to the asymmetries that are observed. Drifts (e.g. $E \times B$ drifts), more complex surface interactions (e.g. chemical erosion), interactions between carbon ions of different charge states, and a realistic spectrum of sputtered carbon energies could all impact the distribution and velocities of the observed carbon ions. A number of additional numerical codes could be brought to bear to provide more insight into the problem, such as ERO for more complex surface interactions [23], and SOLPS-ITER for the inclusion of axisymmetric drifts that could be impacting background flows [24].

\section{v. CONCLUSION}

EMC3-EIRENE modeling was carried out in comparison with MAST experiments with HFS neutral fueling. Good qualitative agreement was found between the deuterium flow structures in modeling and the experimentally measured phenomena in $\mathrm{C}^{2+}$ ions. The use of a 1D model enables us to determine that parallel pressure gradients are the primary drive term in the plasma. Ionization of the puffed neutrals results in an increase in static plasma pressure, that drives deuterium flows away from the fueling perturbation along field lines. Cross field diffusion then causes these flows to slow down. The plasma then influences the carbon ions through an impurity force balance. Work to include carbon ions into the EMC3-EIRENE simulations using an impurity force balance approach from a trace impurity model show that the simulated $\mathrm{C}^{2+}$ ion species qualitatively and quantitatively follows the behavior of the deuterium flows and thus presents strong evidence that it is this pressure driven flow in the main plasma that is behind the $3 \mathrm{D}$ structures observed in the $\mathrm{C}^{2+}$ velocimetry. Future work will focus on addressing the role of background flows, and developing synthetic diagnostic tools to quantitatively benchmark code results against MAST measurements in preparation for the upcoming MAST-U science campaigns.

\section{ACKNOWLEDGMENTS}

This work is funded by the Department of Energy under grant DE- SC0012315 and has been carried out within the framework of the EUROfusion Consortium. The publisher, by accepting this article for publication acknowledges, that the United States Government retains a non-exclusive, paid-up, irrevocable, world-wide license to publish or reproduce the published form of this manuscript, or allow others to do so, for United States Government purposes. It has also received funding from the Euratom research and training program 2014-2018 under grant agreement No 633053 and from the RCUK Energy Program EP/P012450/1. This research was performed using the compute resources and assistance of the UW-Madison Center For High Throughput Computing (CHTC) in the Department of Computer Sciences. The CHTC is supported by UW-Madison, the Advanced Computing Initiative, the Wisconsin Alumni Research Foundation, the Wisconsin Institutes for Discovery, and the National Science Foundation, and is an active member of the Open Science Grid, which is supported by the National Science Foundation and the U.S. Department of Energy's Office of Science. Special thanks to the Culham Centre for Fusion Energy for their hospitality and enthusiasm in enabling this work. 


\section{REFERENCES}

${ }^{1} \mathrm{~T}$ E Evans, R A Moyer, K H Burrell, M E Fenstermacher, I Joseph, A W Leonard, T H Osborne, G D Porter, M J Schaffer, P B Snyder, P R Thomas, J G Watkins, and W P West. Nature Physics, 2(6):419-423, 2006.

${ }^{2}$ Y. Liang, H. R. Koslowski, P. R. Thomas, E. Nardon, B. Alper, P. Andrew, Y. Andrew, G. Arnoux, Y. Baranov, M. Bécoulet, M. Beurskens, T. Biewer, M. Bigi, K. Crombe, E. De La Luna, P. De Vries, W. Fundamenski, S. Gerasimov, C. Giroud, M. P. Gryaznevich, N. Hawkes, S. Hotchin, D. Howell, S. Jachmich, V. Kiptily, L. Moreira, V. Parail, S. D. Pinches, E. Rachlew, and O. Zimmermann. Active control of type-I edge-localized modes with $\mathrm{n}=1$ perturbation fields in the JET tokamak. Physical Review Letters, 98(26), 2007.

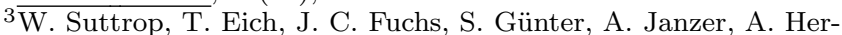
rmann, A. Kallenbach, P. T. Lang, T. Lunt, M. Maraschek, R. M. McDermott, A. Mlynek, T. Pütterich, M. Rott, T. Vierle, E. Wolfrum, Q. Yu, I. Zammuto, and H. Zohm. First observation of edge localized modes mitigation with resonant and nonresonant magnetic perturbations in ASDEX upgrade. Physical Review Letters, 106(22), 2011.

${ }^{4} \mathrm{O}$ Schmitz, T E Evans, M E Fenstermacher, H Frerichs, M W Jakubowski, M J Schaffer, A Wingen, W P West, N H Brooks, K H Burrell, J S deGrassie, Y Feng, K H Finken, P Gohil, M Groth, I Joseph, C J Lasnier, M Lehnen, A W Leonard, S Mordijck, R A Moyer, A Nicolai, T H Osborne, D Reiter, U Samm, K H Spatschek, H Stoschus, B Unterberg, E A Unterberg, J G Watkins, R Wolf, the DIII-D, and TEXTOR Teams. Aspects of three dimensional transport for elm control experiments in iter-similar shape plasmas at low collisionality in diii-d. Plasma Physics and Controlled Fusion, 50(12):124029, 2008.

${ }^{5} \mathrm{O}$ Schmitz, $\mathrm{M}$ Becoulet, $\mathrm{P}$ Cahyna, $\mathrm{T}$ E Evans, Y Feng, H Frerichs, A Loarte, R A Pitts, D Reiser, M E Fenstermacher, and D Harting. Three-dimensional modeling of plasma edge transport and divertor fluxes during application of resonant magnetic perturbations on ITER. Nuclear Fusion, 00:0, 2016.

${ }^{6} \mathrm{H}$ Frerichs, O Schmitz, T Evans, Y Feng, and D Reiter. The pattern of parallel edge plasma flows due to pressure gradients, recycling, and resonant magnetic perturbations in diii-d. Phys. Plasmas, 22:72508, 2015.

${ }^{7}$ Y Feng, H Frerichs, M Kobayashi, and D Reiter. Monte-Carlo fluid approaches to detached plasmas in non-axisymmetric divertor configurations. Plasma Physics and Controlled Fusion, $59(3): 34006,2017$.

${ }^{8}$ S. A. Silburn, J. R. Harrison, J. Howard, K. J. Gibson, H. Meyer, C. A. Michael, and R. M. Sharples. Coherence imaging of scrapeoff-layer and divertor impurity flows in the Mega Amp Spherical Tokamak (invited). Review of Scientific Instruments, 85(11), 2014.

${ }^{9}$ J. Howard. Appl. Opt., 41:197, 2002.

${ }^{10}$ J. Howard, C. Michael, F. Glass, and A.D. Cheetham. Rev. Sci. Instrum., 72:888, 2001.

${ }^{11} \overline{\mathrm{D}}$ Reiter. The EIRENE Code User Manual Version : $11 / 2009$. 2009.
${ }^{12}$ Y Feng, F Sardei, and J Kisslinger. 3D fluid modelling of the edge plasma by means of a Monte Carlo technique. Journal of Nuclear Materials, 266-269(Supplement C):812-818, 1999.

13 H Frerichs, O Schmitz, I Waters, GP Canal, TE Evans, Y Feng, and VA Soukhanovskii. Exploration of magnetic perturbation effects on advanced divertor configurations in nstx-u. Phys. Plasmas, 23:62517, 2016.

${ }^{14}$ D. Harting, D. Reiter, Y. Feng, O. Schmitz, D. Reiser, and $\mathrm{H}$. Frerichs. 3D edge transport studies with EMC3EIRENE for the Dynamic Ergodic Divertor (DED) at TEXTOR. Contributions to Plasma Physics, 48(1-3):99-105, 2008.

15 F Effenberg, Y Feng, O Schmitz, H Frerichs, S A Bozhenkov, H Hölbe, R König, M Krychowiak, T Sunn Pedersen, D Reiter, L Stephey, and W7-X Team. Numerical investigation of plasma edge transport and limiter heat fluxes in Wendelstein 7-X startup plasmas with EMC3-EIRENE. Nuclear Fusion, 57(3):36021, 2017.

${ }^{16}$ W Zhang, V Bobkov, T Lunt, J.-M. Noterdaeme, D Coster, R Bilato, P Jacquet, D Brida, Y Feng, E Wolfrum, L Guimarais, and the ASDEX Upgrade Team. 3D simulations of gas puff effects on edge density and ICRF coupling in ASDEX Upgrade. Nuclear Fusion, 56(3):36007, 2016.

17 Shuyu Dai, M. Kobayashi, G. Kawamura, S. Morita, H. M. Zhang, T. Oishi, Y. Feng, D. Z. Wang, and Y. Suzuki. EMC3EIRENE modelling of edge impurity transport in the stochastic layer of the large helical device compared with extreme ultraviolet emission measurements. Nuclear Fusion, 56(6), 2016.

${ }^{18}$ T. Oishi, S. Morita, S.Y. Dai, M. Kobayashi, G. Kawamura, X.L. Huang, H.M. Zhang, Y. Liu, and M. Goto. Observation of carbon impurity flow in the edge stochastic magnetic field layer of Large Helical Device and its impact on the edge impurity control. Nuclear Fusion, 58(1), 2018.

19 A. R. Field, P. G. Carolan, N. J. Conway, G. F. Counsell, G. Cunningham, P. Helander, H. Meyer, D. Taylor, M. R. Tournianski, and M. J. Walsh. The influence of gas fuelling location on $\mathrm{H}-$ mode access in the MAST spherical tokamak. Plasma Physics and Controlled Fusion, 46(6):981-1007, 2004.

${ }^{20}$ P. Stangeby. Plasma Bound. Magn. Fusion Devices. Institute of Physics Publishing, 2000.

${ }^{21}$ M. Carr, A. Meakins, A. Baciero, M. Bernert, A. Callarelli, A. Field, C. Giroud, J. Harrison, N. Hawkes, S. Henderson, and B. Lipschultz. Towards integrated data analysis of divertor diagnostics with ray-tracing. In 44th EPS Conference on Plasma Physics, Belfast, Northern Ireland UK, 2017.

${ }^{22}$ H. Frerichs, F. Effenberg, Y. Feng, O. Schmitz, L. Stephey, D. Reiter, and P. Börner. Reconstruction of recycling flux from synthetic camera images, evaluated for the Wendelstein 7-X startup limiter. Nuclear Fusion, 57(12), 2017.

${ }^{23}$ A Kirschner, P Wienhold, V Philipps, J P Coad, A Huber, and U Samm. Modelling of carbon transport in fusion devices: evidence of enhanced re-erosion of in-situ re-deposited carbon. Journal of Nuclear Materials, 328(1):62-66, 2004.

${ }^{24}$ S. Wiesen, D. Reiter, V. Kotov, M. Baelmans, W. Dekeyser, A. S. Kukushkin, S. W. Lisgo, R. A. Pitts, V. Rozhansky, G. Saibene, I. Veselova, and S. Voskoboynikov. The new SOLPS-ITER code package. Journal of Nuclear Materials, 463:480-484, 2015. 